dimensionality of the neural space and the dimensionality of the muscle space. Such disparities lead to redundancy, with different neural patterns causing the same muscle activity while others do not. Kaufman et al. ${ }^{1}$ propose that the redundant neural dimensions are tightly controlled for the specific purpose of motor preparation. In a contrasting example of the exploitation of redundancy in the motor system, the 'uncontrolled manifold' hypothesis suggests that redundant biomechanical dimensions are not controlled and are allowed to vary almost randomly if they do not affect performance ${ }^{13}$.

One of the striking findings of the analysis is that the entire theory is consistent with a linear or near-linear model relating cortical neural population activity to motor control. The anatomy and biomechanical properties of the primate skeletomotor system are notoriously complex and nonlinear. Any motor controller designed on first principles derived from physics that would attempt to match the performance of the biological motor system would have to be equally complex and nonlinear. Nevertheless, as with all studies of complicated nonlinear systems, it is often best to start by determining how far we can go with a much simpler linear model. It is surprising that the output-potent and output-null dimensions can be extracted by a linear regression between neural activity and muscle activity and that they are truly orthogonal in the sense predicted by linear algebra. This did not have to be the case: the two dimensions could have been twisted through the space of possible neural activations in intricate ways. And yet the output-null dimension can be approximately described by a hyperplane that is the null space of a linear (matrix) transformation between cortical activity and muscle activity. This is reminiscent of the success of the population-vector analysis, for which the relation between motor cortical firing and movement kinematics is approximately linear ${ }^{14}$. This near linearity might indicate that cortical control processes are a "good enough" solution ${ }^{15}$ that provides subcortical motor circuits with enough information to initiate an approximation of the desired action, whereas the latter use feedforward and feedback signals to deal with motor errors and the nonlinearities of the peripheral skeletomotor apparatus. Moreover, this seeming simplicity might reflect the influence of evolutionary processes that acted to optimize both the peripheral motor apparatus and its central neural controller as they coevolved over millions of years.

The mechanism proposed by Kaufman et al. ${ }^{1}$ is necessarily a property of populations of neurons, rather than individual neurons, and it represents a very interesting example of the emerging role of theories that are able to predict behavior from simultaneously recorded neural populations rather than from single cells. The existence and role of the output-null dimensions would not have been detectable from singlecell recordings. Indeed, the authors point out that the same cell may participate in both the outputpotent and output-null dimensions at different times, even though in the latter case its potential effect on muscles must be cancelled out by the activity of other neurons in the population. As multielectrode recordings become more and more prevalent, the availability and importance of population-based theories of neural encoding will become increasingly evident. Just as multiple visual images can be overlaid and perceived simultaneously, so populations of neurons have tremendous power to represent more than one perception or movement simultaneously. Perhaps this is how we can sit still without acting out our every motor whim whenever we imagine doing something, and how the hunting tiger can crouch motionless while preparing to pounce.

\section{COMPETING FINANCIAL INTERESTS}

The authors declare no competing financial interests.

1. Kaufman, M.T., Churchland, M.M., Ryu, S.I. \& Shenoy, K.V. Nat. Neurosci. 17, 440-448 (2014).

2. Hétu, S. et al. Neurosci. Biobehav. Rev. 37, 930-949 (2013).

3. Bremner, L.R. \& Andersen, R.A. Neuron 75, 342-351 (2012)

4. Buch, E.R., Brasted, P.J. \& Wise, S.P. Exp. Brain Res. 169, 69-84 (2006).

5. Rizzolatti, G. \& Sinigaglia, C. Nat. Rev. Neurosci. 11, 264-274 (2010).

6. Vigneswaran, G., Philipp, R., Lemon, R.N. \& Kraskov, A. Curr. Biol. 23, 236-243 (2013).

7. Hishikawa, Y. \& Shimizu, T. Adv. Neurol. 67, 245-271 (1995).

8. Evinger, C., Kanako, C.R. \& Fuchs, A.F. J. Neurophysiol. 47, 827-844 (1982).

9. Kaufman, M.T. et al. J. Neurophysiol. 104, 799-810 (2010).

10. Kaufman, M.T., Churchland, M.M. \& Shenoy, K.V. J. Neurophysiol. 110, 817-825 (2013).

11. Cohen, O., Sherman, E., Zinger, N., Perlmutter, S. \& Prut, Y. Curr. Opin. Neurobiol. 20, 696-703 (2010).

12. Afshar, A. et al. Neuron 71, 555-564 (2011).

13. Scholz, J.P. \& Schöner, G. Exp. Brain Res. 126, 289-306 (1999).

14. Georgopoulos, A.P., Kettner, R.E. \& Schwartz, A.B. J. Neurosci. 8, 2928-2937 (2008).

15. Partridge, L.D. Am. J. Physiol. 242, R172-R177 (1982).

\title{
Oxytocin for all senses
}

Deprivation of one sensory modality is known to diminish cortical responses in the corresponding sensory cortex as well as alter neuronal responses in unrelated sensory cortices. Yet little is known about what drives this cross-modal plasticity. On page 391, Zheng and colleagues explore the effects of early unimodal sensory deprivation in multiple sensory cortical regions in mice and report that oxytocin, a neuropeptide important for lactation, parturition, social and emotional behaviors, is critical for cross-modal cortical plasticity.

Zheng and colleagues subjected mice to sensory deprivation starting from birth by either removing their whiskers or raising them in the dark. Each type of deprivation decreased spontaneous excitatory synaptic transmission and sensory stimulation-evoked responses in both primary somatosensory (S1) and primary visual (V1) cortex but not in higher order cortical regions such as the prefrontal cortex. This was accompanied by a decrease in oxytocin levels in the sensory cortex affected by the sensory deprivation. Whisker removal or dark rearing also reduced oxytocin production and expression in the hypothalamus. Oxytocin injection into S1 not only increased excitatory synaptic transmission in both S1 and V1 but also reversed the cross-modal effects of sensory deprivation in these areas. To complement their findings from sensory deprivation, Zheng et al. then reared mice in a sensory-

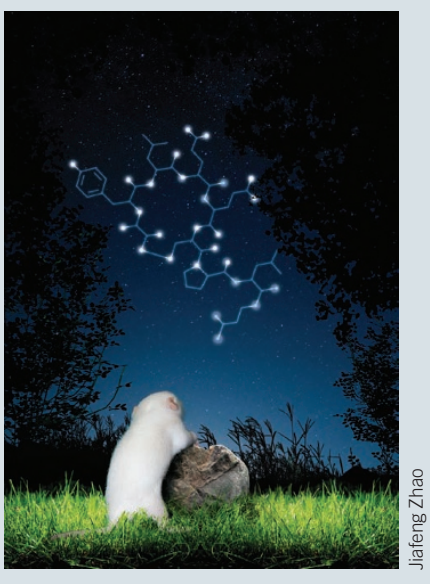
enriched environment. This manipulation led to increased oxytocin production in the hypothalamus, higher levels of oxytocin expression in S1 and V1, and enhanced neuronal responses in these cortical areas. Sensory enrichment also rescued the effects of sensory deprivation, similar to the treatment with exogenous oxytocin.

These findings reveal a critical function for oxytocin in activity-dependent cortical development and cortical plasticity. 